Article

\title{
Identification of Fatigue Damage Stages in Polymer Composite Materials by using Acoustic Emission: Approach and Perspec- tives
}

\author{
Oleg Bashkov 1,2, Anton Bryansky 1,2, Timofey Efimov ${ }^{2}$ and Roman Romashko ${ }^{2,3, *}$ \\ 1 Komsomolsk-na-Amure State University, Russia; bashkov@knastu.ru \\ 2 Institute of Automation and Control Processes FEB RAS, Russia; romashko@iacp.dvo.ru \\ 3 Far-Eastern Federal University, Russia \\ * Correspondence: romashko@iacp.dvo.ru; Tel.: +7-423-2310439
}

\begin{abstract}
The work is devoted to the study of the mechanisms of damage accumulation in a polymer composite material (PCM) during fatigue loading. Mechanical testing of a fiberglass sample was carried out by cyclic tension accompanied by registration of acoustic emission (AE). For the recorded AE signals, the Fourier spectra were calculated and used for clustering with Kohonen self-organizing map. Relations between clusters and types of damage in the PCM structure were established. The analysis of the peak frequencies of the Daubechies D14-wavelet components of AE signals was carried out. Obtained results has allows one to describe the processes of destruction in the PCM sample. It has been established that, on the base of local formation of microdamages in the matrix and the fracture of the fibers detected during recording of the $\mathrm{AE}$ data, it is possible to predict the destruction of the polymer composite material, while the beginning of a material destruction can be registered if the damage identified as an adhesion failure is observed. Perspectives of application of adaptive fiber-optic AE sensors for structural monitoring of PCMs on the base of preliminary experimental results are considered and discussed.
\end{abstract}

Keywords: PCM; fiberglass; destruction; cyclic load,; fatigue damage; AE; clusterization; fiber-optical sensors

\section{Introduction}

Acoustic emission (AE) - one of the non-destructive testing (NDT) techniques, which allows to obtain information on the processes of structure damage in polymer composite materials (PCM) during mechanical deformation, when the use of other NDT methods is restricted or complicated [1,2]. Advanced techniques for processing AE data based on multivariate analysis make it possible to classify similar signals into groups and characterize their sources [3].

During statistical analysis of AE signals the following parameters are under estimating: amplitude, energy, pike and median frequencies [4-8]. However the data obtained with use of these parameters is not enough to characterize AE sources in case of considerable number of AE events [1]. Thus, the frequency spectra of detected signal is to be taken into account in order to assess effectively the source properties that generated $\mathrm{AE}$ wave $[3,9,10]$.

One of the methods for statistical analysis of AE data represented by numerical series is the clustering. If the information about parameters being registered is unavailable the unsupervised methods are to be used. The last include the $k$-means algorithm and the selforganizing Kohonen maps (SOM) [7,11-13]. In order to solve the clustering problem the complex analysis of amplitudes and frequency components of AE signals have been successfully applied in works [12,14] while energy and peak frequency as a reference 
parameters were used in the work for the case of static loading [1]. In our research we apply statistical analysis of the AE signal amplitude and frequency components for the case of a cycling load of specimen. The obtained results demonstrate the possibility of identifying the stages of damage accumulation during cyclic testing of a PCM sample. Experiments were carried out at the 50\% from maximal load.

\section{Materials and Methods}

The object of this research was dog-bone shaped specimens cut from a FGRP plate with a rectangular cross section of $10 \times 10 \mathrm{~mm}^{2}$. The FGRP plate was made by vacuum infusion process using DION 9300 FR epoxy vinyl ester binder and 46 layers of T11-GVS9 glass fabric.

Mechanical tests were carried out on a servo-hydraulic testing machine using the cyclic tension method. The value of the stress cycle amplitude was chosen equal to half of the breaking load and amounted to $300 \mathrm{MPa}$.

Registration of AE signals was carried out by means of the software-hardware complex AE-2.1-Pro using the Globaltest GT301 wideband sensor (50 - $550 \mathrm{kHz}$ ) [15]. The registered AE signals were processed with use of MATLAB-based home-made software according to the scheme shown in Figure 1.

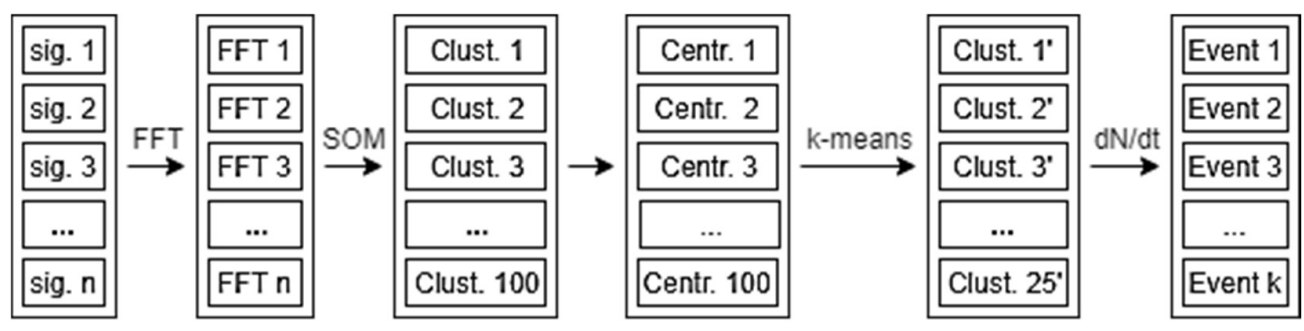

Figure 1. Registered AE signals processing scheme.

In this work, data clustering was implemented by the two-level approach [10]: at first level, the cluster centroids is extracted from FFT spectra with using Kohonen self-organizing map (SOM), while, at the second level, the obtained centroids are grouped by using the $k$-means algorithm.

According to the results of works [6,9,14], the frequency range $24-450 \mathrm{kHz}$ as most informative part of AE signals Fourier spectra was taken for further analysis. The obtained spectra were fed to the input of a SOM. Amount of output clusters (100 pieces) was selected empirically when assessing the reproducibility of clustering results, including another types of mechanical tests [16]. Also, such amount of clusters makes it possible to distinguish the damage of a mixed nature in the material and noise [11].

Further analysis involves combining clusters by the similarity of their centroids. However, a small amount of data of the 100 centroids and a large number of their parameters of the 1352 values of each spectrum leads to unsatisfactory clustering results [3]. Reducing the data dimension, and in this case, reducing the centroids spectra resolution, allows to increase the efficiency of using $k$-means and other algorithms [2]. The centroid resolution was reduced down to 86 spectral lines with a step of $5 \mathrm{kHz}$. Amount of the output clusters was taken to be 25 considering 5 possible types of damage and their combinations. Similar output clusters were combined manually.

The accumulation diagrams of each cluster were analyzed according to the rate of their accumulation. The activity of defects developing in PCM was chosen as informative parameter in the clustering analysis [17]. The source of damage was considered to be active when the AE activity within the cluster exceed the threshold value of $2 \mathrm{~s}^{-1}[18,19]$.

The coefficients of the wavelet decomposition can also be used to characterize the AE signals $[7,19]$. Earlier, the Daubechies wavelet decomposition of the $14^{\text {th }}$ order for the registered AE data analysis at three-point static bending of fiberglass specimens analysis was 
used and approved in work [20]. According to the peak frequencies which characterize the different types of damage, the band of each level of decomposition was associated with certain type of damage. Taking into account the influence of the path propagation length of the acoustic wave on the registered signal peak frequency [6], the spread of the peak frequencies of each level can be used to estimate the local or global scale of the resulting damage in the material volume.

\section{Results and discussion}

\subsection{Two-level clustering (SOM and k-means)}

As a result of acoustic emission signals processing according to the scheme shown in Fig. 1, 18 clusters were obtained. The characteristics of the clusters which correspond to certain type of damage were carried out on the base of the peak frequencies values. In works $[3,4,7,14]$ it was found that frequency range $30-150 \mathrm{kHz}$ corresponds to matrix microdamage, frequency range $130-220 \mathrm{kHz}$ corresponds to delamination, while frequency ranges $230-310 \mathrm{kHz}, 180-290 \mathrm{kHz}$ and over $380 \mathrm{kHz}$ corresponds to debonding, slipping and breaking of fibers, respectively. In this study, in the frequency range of the matrix microdamage, 4 peaks in the sub-ranges $25-40,55-70,80$ and $100-105 \mathrm{kHz}$ can be resolved.

According to the volume of local destructions, three scales of the matrix damages a were discriminated. Local damages of the matrix in areas with dimensions not exceeding the cross-section of several reinforcing fibers were attributed to the scale 1 . Shear damages of the polymer matrix in the volume of one or several strands of glass fibers were attributed to the scale 2. Chips and breaks of the matrix in a larger volume which proceed without delamination were attributed to the scale 3.

For the specimens made of pure binder by three-point bending being under test, at the moment of sliding of the specimen along the supports, AE signals with a peak frequency of about $68 \mathrm{kHz}$ were registered, while for tension, at the moment of insertion of the grips of the testing machine into the surface of the binder and its damage, AE signals with peak frequencies of 27 and $67 \mathrm{kHz}$ were registered. Also, at PCM three-point bend test, matrix damages are characterized by a peak frequencies in the range of $24-110 \mathrm{kHz}$. Based on these data, frequencies of $30-70 \mathrm{kHz}$ and $80 \mathrm{kHz}$ was assumed as characterizing the matrix damage of scale 1 and scale 2 (shear and compression microdamages), the frequency $100 \mathrm{kHz}$ relates to the matrix damage of scale 3 (chips, breaks), and frequencies in the range $130-150 \mathrm{kHz}$ indicates to matrix damage of delamination type. The frequencies and the corresponding to them types of forming damages assumed in this work are summarized in the Table 1.

Table 1. Frequencies ranges and corresponding to them types of damage

\begin{tabular}{cc}
\hline Frequencies, $\mathbf{k H z}$ & Type of damage \\
\hline $30-70,80$ and 100 & Matrix damages of scale 1, 2 and 3 respectively \\
$130-150$ & Critical matrix damage (interlayer damage) \\
$150-220$ & Delamination \\
$180-260$ & Fibers slipping \\
$270-310$ & Fibers debonding \\
$360-400$ & Fibers failure \\
\hline
\end{tabular}

The peak frequencies of the AE signal spectra clusters obtained at the second level of clustering (reworked clusters, $\mathrm{RC}$ ) are given in the Table 2, where peak frequencies with lower magnitude are indicated in parentheses. It is worth to noting that some deformations are accompanied by complex superposition of various damages which results in high AE activity represented by of sequential stream of signals not resolved in time [12]. Such complicated cases are covered by the cluster "Mixed damages" (RC3).

Table 2. Reworked clusters description 


\begin{tabular}{ccc}
\hline Cluster & Frequencies, $\mathbf{~ k H z}$ & Description \\
\hline RC1 & $35,100,380-400(60,80)$ & Matrix and fibers combined damage \\
RC2 & $370-400$ & Fibers failure \\
RC3 & $35-400$ & Mixed damages \\
RC4 & 35 & Matrix damage, scale 1 \\
RC5 & $170-260,300,360-400(35-130)$ & Debonding, slipping and fibers failure \\
RC6 & $300-310,360-380(240-250)$ & Debonding and fibers failure \\
RC7 & $30,80(60,100)$ & Matrix damage, scale 1 prevails \\
RC8 & $30-60(80-100)$ & Matrix damage, scale 2 prevails \\
RC9 & 100 & Matrix damage, scale 3 \\
RC10 & $60-80$ & Matrix damage, scale 2 \\
RC11 & $100(30-80)$ & Matrix damage, scale 3 prevails \\
RC12 & $60-80,380(30,100)$ & Combined damage of matrix (scale 2) and fibers \\
RC13 & $200-240(270-300,360-400)$ & Delamination forming, fibers failure \\
RC14 & $170-180,360-380$ & Slipping and fibers failure \\
RC15 & 80 & Matrix damage, scale 2 \\
RC16 & $180(30-130,380)$ & Interlayer damage (delamination) \\
RC17 & $30,80-130,150$ & Critical matrix damage \\
RC18 & $100,360-380$ & Matrix scale of 3 and fibers combined damage \\
\hline
\end{tabular}

For determining of the destruction process stages at the cycle loading conditions, it is important to take into account the sequence and collective action of the forming defects. Therefore, for each cluster, the time intervals within those the rate of damage registration events exceeds the threshold level (two events per second) were determined. These periods of AE activity for each cluster are shown in Fig.2.

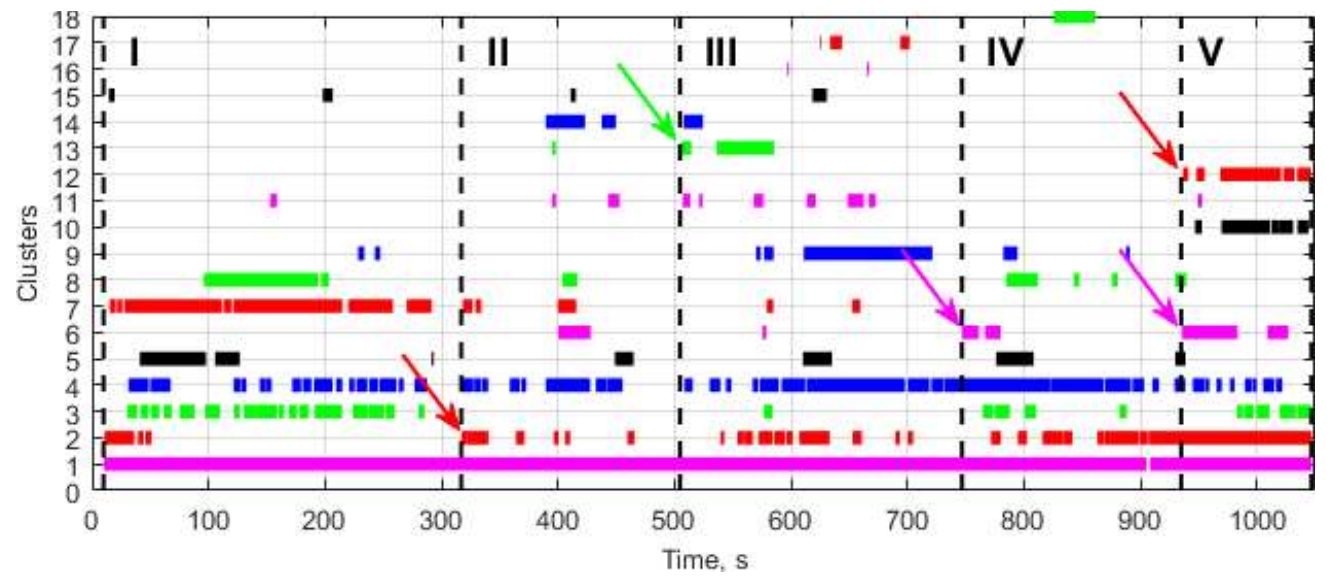

Figure. 2. Periods of exceeding the intensity threshold of each cluster accumulation.

First, the periods of only matrix damage of scale 1 and fibers failure were identified, which were taken as the boundaries of the stages [17]. All period of PCM specimens cycle testing until its failure was sub-divided into 5 stages. The selection of stages was carried out on the base of test results for five specimens included in a representative sampling. Fig. 2 shows the results of data analysis for one of PCM specimens from the representative sampling, which made it possible to identify the stages common for whole sampling.

All stages of material destruction are characterized by formation of damages of two types: (i) combined damages in the matrix and fibers and (ii) individual damages in the matrix. In addition, it is worth highlighting the accumulation of individual damage in glass fibers which becomes more intense from stage to stage.

Stage I. Mostly characterized by matrix damages at the scale 1 or 2 . Most likely, the fibers failure at the early stage is caused by material damaging in the grips of the testing 
machine. This can be eliminated by applying other grips during testing which prevent damaging or by selecting and filtering out the group of signals generated by sources located in the grips.

Stage II. Combined damage of both matrix and fibers are formed. An interlayer crack is initiated, which results in matrix damages at scale 3, as well as more complicated and intense fiber damages.

Stage III. Formation of the combined matrix damages and fibers failure, the further growth of interlayer cracks in the material. Starting from the middle of the stage, periodic critical matrix damages occur, and the rate of matrix damages formation at the scale 3 is increased significantly.

Stage IV. The process of formation of combined matrix damages and fibers failure becomes uniform and accompanied by periodic registration of mixed material damages. On this stage, the matrix damages are represented on the scale 1, while the mechanism of fibers damage is mainly represented only by fibers fracture.

Stage V. The matrix damages in combination with fibers failure occur at the scale 3. Mixed damage of material, polymer matrix damages at the scales 1 and 2 (with prevails of the scale 2) are observed.

\subsection{Wavelet decomposition}

The assessment of the local or global scale of formed damage in the material was done by the spread of the peak frequencies of each decomposition level. Considering the sampling frequency of the registering equipment equal to $5 \mathrm{MHz}$ and the number of used decomposition levels equal to seven, the obtained frequency bands as well as the correspondence of damage types to decomposition levels are given in the Table 3.

Table 3. Frequency bands of the decomposition levels

\begin{tabular}{ccc}
\hline Level & Frequency band, $\mathbf{k H z}$ & Damage type \\
\hline 1 & $1250-2500$ & - \\
2 & $625-1250$ & - \\
3 & $312,5-625$ & Fiber failure \\
4 & $156,25-312,5$ & Adhesion loss (delamination, fibers debonding) \\
5 & $78,13-156,25$ & Critical matrix damage \\
6 & $39,06-78,13$ & Matrix major damage \\
7 & $19,53-39,06$ & Matrix microdamage \\
\hline
\end{tabular}

Since the frequency ranges of decomposition levels 3, 4 and 5, according to the performed classification, correspond to several types of damage at once, the median values and standard deviation of the peak frequencies were calculated for analysis (Fig. 3). The predominant type of PCM damage was determined by the median value of the peak amplitude. The scale of the resulting damage was estimated from the standard deviation of the values. The stage boundaries are taken from the analysis of the clusters accumulation.

At the first stage of material destruction, a matrix damages occur locally at the scale 2 (microscale) with a tendency of scale reduction. Damages associated with glass fibers are mainly represented by adhesion loss to the binder. The scale of matrix damage at the second stage begins to take on a global character. The intensity of the fibers debonding decreases and fibers begin to break in the material volume. At the third stage, matrix damages are formed locally but at larger scale. Fibers failure again acquires in a local character, and damages of the type of adhesion loss are formed chaotically. At the fourth stage, an increase in the scale of matrix damage with their local formation persists, active global debonding and slipping of fibers with their subsequent fracture continues. The fifth stage is characterized by global critical material damage and sample destruction. 


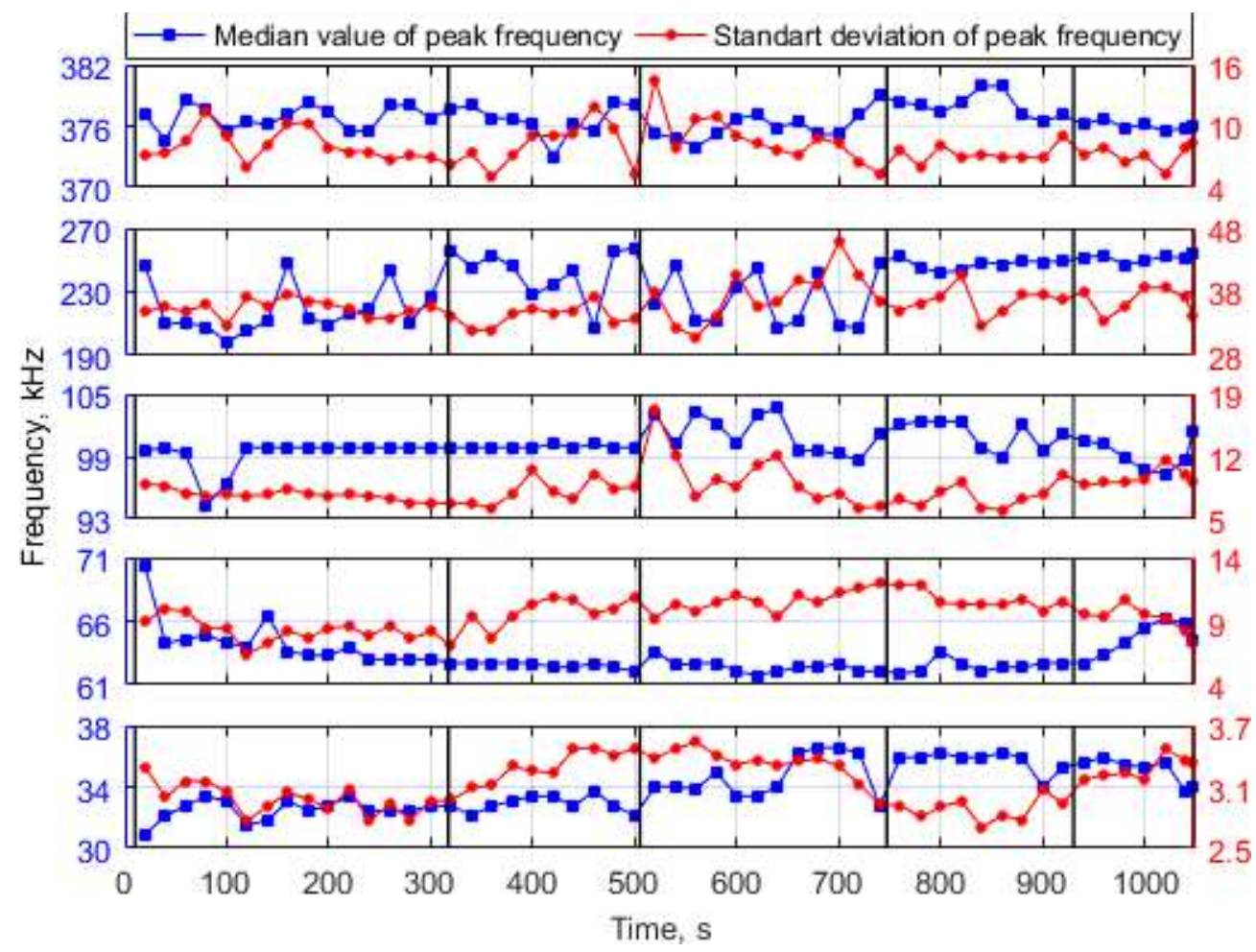

Figure. 3. Diagrams of changes in the median value of the peak frequencies and their standard deviation over time for decomposition levels from 3 to 7 (from top to bottom).

\section{Application of distributed fiber-optical AE sensors for PCM monitoring}

In the study, the following problems of the current level of the hardware base for $\mathrm{AE}$ registration were revealed.

1. The complex structure of the polymer composite, namely the effect of stress transfer by the matrix on the volume of the reinforcing filler, leads to the distribution of damage in the material. This problem can be characterized as the formation of local structural damage, leading to a redistribution of the stress field and the likelihood of formation of associated damage located at a sufficient distance from the original [21-23].

2. A large number of reinforcing fabrics used in the manufacture of PCMs have an anisotropic structure due to differences in the content of fibers of the weft and the base of the fabric, which causes the inhomogeneity in the medium of acoustic signals propagation [24-26]. Special techniques for sequential packing of layers characterized by the multidirectional axis of reinforcement only partially solve this problem, since full isotropy in all directions is not achieved [27].

3. A high content of filler in the composite which is required for providing high strength values determine a large area of phase boundaries. Additionally, due to multilayer structure of the composite, this feature leads to a scattering of high-frequency components of acoustic wave at the "polymer matrix - fiber" interface [28].

Global damage of the structure revealed by visual observation of the PCM sample during cyclic tensile testing with load amplitude up to $0,6 \sigma_{\mathrm{B}}$, was confirmed by analyzing the parameters of the recorded AE. A large number (18) of resulting output clusters of frequency representations of AE signals is due to a variety of damage scales. However, for extended structures, it can be related to the remoteness of the AE source from the transducer that can distort the spectrum of the AE signals due to the dispersion of sound speed in plates or rods, where the longitudinal waves are transformed into Lamb or Porkhhammer waves [29, 30]. 
Larger the size of an object under study, larger the required number of AE transducers. In this case, the inhomogeneity of the properties of PCM will inevitably affect the results of signal analysis recorded by the AE transducers located at different distances from the radiation source. Moreover, significant uncertainties in the map of anisotropic properties distribution in the material will make additional correspond influence onto AE signals.

In turn, fiber-optic acoustic sensors (FOS) with distributed sensitivity represent one of the most promising means for implementing a system for monitoring a PCM with a complex structure [31-33]. Sensing optical fibers can be embedded in PCMs during their manufacturing [34-36]. This makes it possible to ensure the coverage of the entire surface of the objects by transducers sensors, which is required for monitoring the state of structural elements. FOS placing over the object surface makes it possible to reduce the influence of the acoustic signal attenuation and its distortion due to damage accumulating in the material. Moreover, opening possibility to register an acoustic waves with wider front will provide information about distribution of acoustic energy in the object under control.

To register AE signals in the PCM plate, we used an FOS based on the use of an adaptive laser holographic interferometer, which key element is a dynamic hologram continuously recorded in a photorefractive crystal in scheme of two-wave mixing [37]. This type of interferometer allows maintaining high sensitivity and eliminating the influence of low-frequency mechanical vibrations, fluctuations of temperature or pressure and other noisy factors on the output signal. Complete description of the adaptive interferometer is given in the papers [38-40]. Multimode optical fibers with a core diameter of $62.5 \mu \mathrm{m}$ were used as sensitive elements of the interferometer. The fibers were built into the PCM between layers of fiberglass during the manufacturing process by the method of vacuum infusion. The PCM plate was manufactured with lateral dimensions of $500 \mathrm{~mm} \times 500 \mathrm{~mm}$ and thickness of $9 \mathrm{~mm}$. Nine optical fibers (three along and three across the plate) were embedded in the PCM plate. In this work, we present the results obtained using a fiber that was located parallel to one of the sides of the plate at the distance of $100 \mathrm{~mm}$ from the edge.

The simulation of the excitation of AE waves in the PCM plate was performed by breaking a pencil lead with a diameter of $0,5 \mathrm{~mm}$ and hardness $\mathrm{H}$ (source of Hsu-Nielsen). The source was located at a distance of $300 \mathrm{~mm}$ from the optical fiber. AE signals were recorded not only by FOS but also by PZT which was installed on a plate above the optical fiber in its middle part. A photo of the plate with the arrangement of FOS, PZT and the AE source location is shown in Fig. 4.

The AE signals recorded at the moments of breaking the pencil lead at the surface of the PCM plate and their Fourier spectra are shown in Fig. 5. As seen, the waveforms recorded by FOS and PZT differ from each other. The FOS signal decays more slowly than the PZT signal. This is due to the distributed nature of the FOS which detect the acoustic wave, being moving radially from the source, which at first reaching the center of the built-in part of the optical fiber and then gradually spreading to its edges. The frequency response of the FOS is linear in contrast to the response of the resonant PZT. Due to the fact that the absorption of sound waves increases with increasing frequency, the Fourier spectrum for FOS has a pronounced exponential decrease of magnitude at higher frequencies. For the particular FOS, the magnitude for frequencies above $50 \mathrm{kHz}$ is weakly noticeable. In turn, the spectrum of the signal recorded by the PZT contains harmonics up to 400 $\mathrm{kHz}$. 


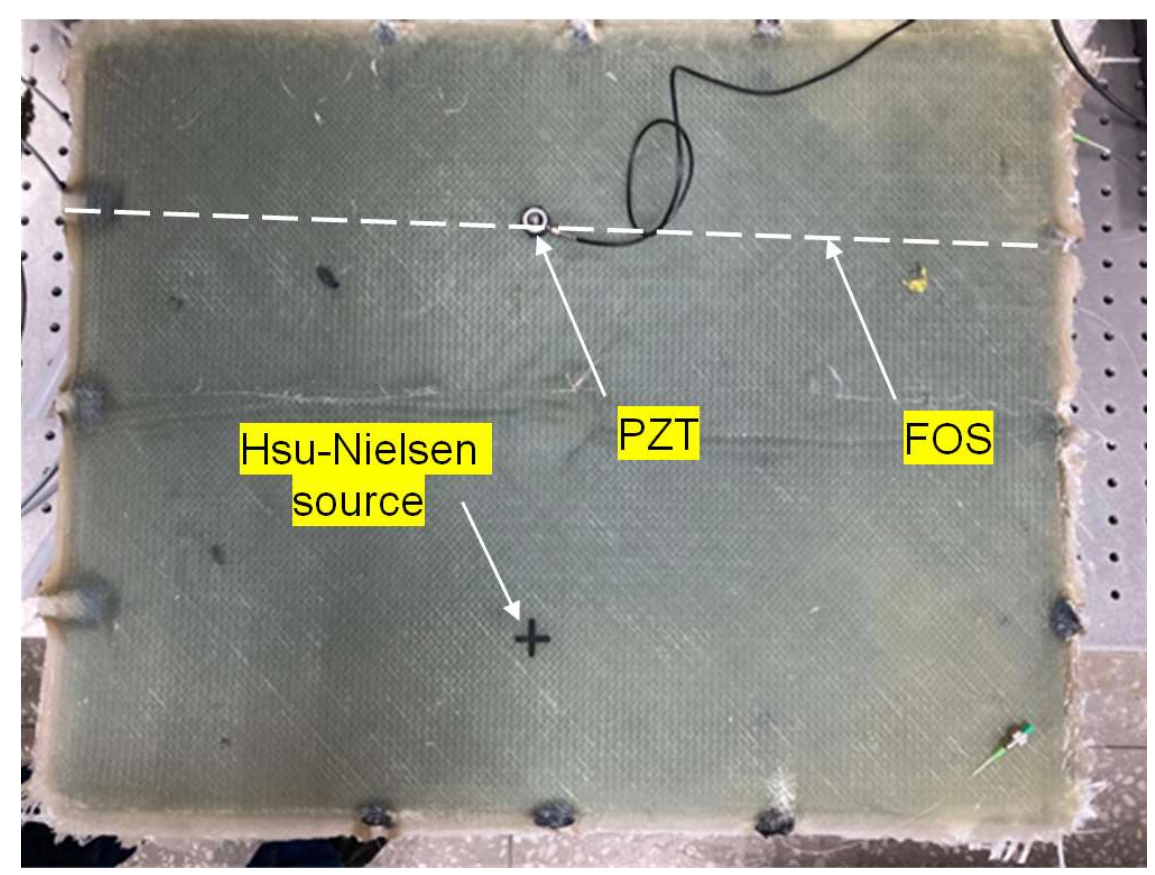

Figure. 4. Top-view of the PCM plate with embedded FOSs, placed on its surface PZT and location of Hsu-Nielsen source.

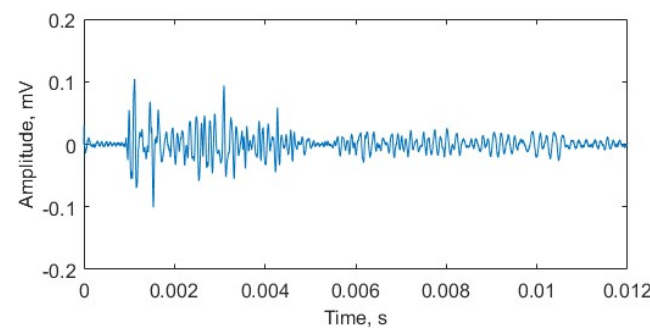

(a)

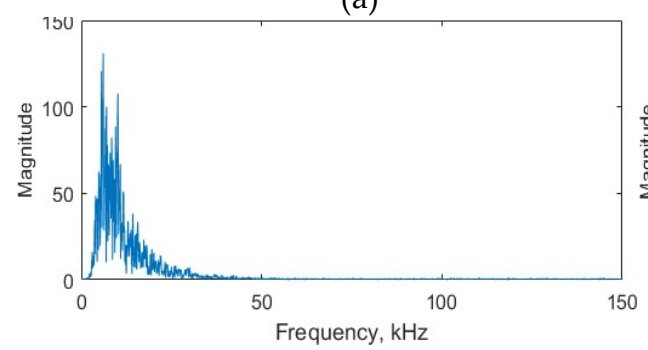

(c)

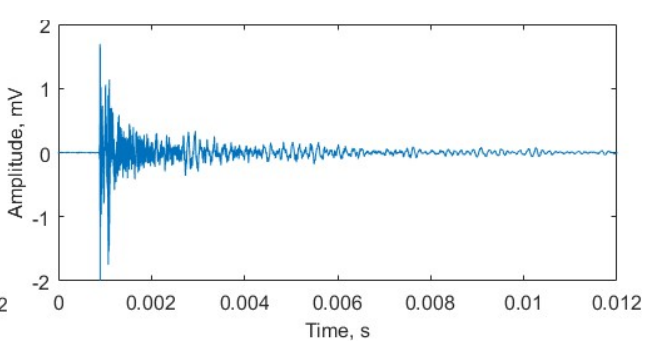

(b)

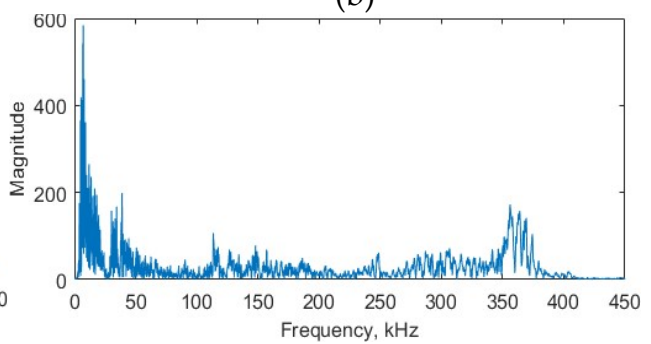

(d)

Figure. 5. AE signals $(a, b)$ and their Fourier spectra $(c, d)$ obtained with use of FOS $(a, c)$ and PZT $(\mathrm{b}, \mathrm{d})$.

In spite of limited spectral range of FOS sensitivity in comparison with PZT, it is worth to note that, for monitoring most of extended objects, PZTs with characteristic frequency not exceeding $60 \mathrm{kHz}$ are usually applied. This makes promising use of optical fibers as distributed sensing elements in SHM systems applied for monitoring the polymer composite materials.

\section{Conclusion}

The results obtained demonstrate the possibility of identifying the stages of damage accumulation during cyclic tensile testing of PCM specimens with a load amplitude up to $0,6 \sigma$ в. The technique is based on Fourier cluster analysis of the spectra of AE signals 
recorded during the tests and the identification of stages of PCM destruction, which correspond to modern knowledge on the essence and nature of destruction of composite materials.

Based on the analysis of the clusters activity and the their correspondence to the type of PCM damage, 5 stages of PCM destruction from the initial damage of the matrix up to combined damage of both matrix and fibers were identified. It was found that the achievement of material state prior to its destruction can be detected by the local formation of microdamages in the matrix and the fracture of a part of the fibers.

A distributed fiber-optic sensor based on an adaptive laser holographic interferometer was tested for recording AE signals upon excitation of acoustic waves in the PCM plate by the Hsu-Nielsen simulator. The recorded signals are characterized by an informative frequency range of the Fourier spectrum up to $50 \mathrm{kHz}$. To adapt the specified range to the results obtained in this work, it is necessary to carry out additional studies with the registration of AE signals by fiber-optic sensors embedded into the PCM being destroyed under the influence of various types of loads. This will allow to apply the damage identification technique for new types of fiber-optic sensors used for structural health monitoring and non-destructive online testing of different PCM-based construction during their exploitation.

Author Contributions: Conceptualization, O.B. and R.R.; methodology, O.B.; formal analysis, A.B. and O.B.; investigation, A.B. and T.E.; writing-original draft preparation, O.B and A.B.; writingreview and editing, R.R.; project administration, R.R. All authors have read and agreed to the published version of the manuscript.

Funding: This research was funded by RUSSIAN SCIENCE FOUNDATION, grant number 21-1900896.

Conflicts of Interest: The authors declare no conflict of interest.

\section{References}

1. Fengming, Y.U.; Okabe, Y.; Qi, W.U.; Shigeta, N. Damage type identification based on acoustic emission detection using a fiberoptic sensor in carbon fiber reinforced plastic laminates. JAE 2016, 33, 543-550.

2. Enoki, M.; Muto, Y.; Shiraiwa, T. Evaluation of deformation behavior in LPSO-magnesium alloys by AE clustering and inverse analysis. JAE 2016, 33, 145-150.

3. Sause, M.G.R. Acoustic emission source identification in large scale fibre reinforced composites. JAE 2016, 33, 441-450.

4. Willems, F.; Benz, J.; Bonten, C. Detecting the critical strain of fiber reinforced plastics by means of acoustic emission analysis. JAE 2016, 33, 525-534.

5. Hill, E.V.K.; Rovik, C.L. In-flight fatigue crack growth monitoring in a Cessna T-303 Crusader vertical tail. JAE 2013, $31,19-36$.

6. Hamam, Z.; Godin, N.; Fusco, C.; Monnier, T. Modelling of fiber break as acoustic emission source in single fiber fragmentation test: comparison with experimental results. JAE 2018, 35, 1-12.

7. Gutkin, R.; Green, C.J.; Vangrattanachai, S.; Pinho, S.T.; Robinson, P.; Curtis, P.T.. On acoustic emission for failure investigation in CFRP: Pattern recognition and peak frequency analyses. Mech Syst Signal Process 2011, 25(4), 1393-1407, doi: 10.1016/j.ymssp.2010.11.014.

8. Bashkov, O.V.; Protsenko, A.E.; Bryanskii, A.A.; Romashko, R.V. Diagnostics of polymer composite materials and analysis of their production technology by using the method of acoustic emission. Mech Compos Mater 2017, 53(4), 533-540, doi: 10.1007/s11029-017-9683-7

9. Sause, M.G.R. On use of signal features for acoustic emission source identification in fibre-reinforced composites. JAE 2018, 35, $1-12$.

10. De Oliveira, R.; Marques, A.T. Health monitoring of FRP using acoustic emission and artificial neural networks. Comput Struct 2008, 86(3-5), 367-373, doi: 10.1016/j.compstruc.2007.02.015.

11. Hill, E.V.K.; Foti, C.J.; Leung, N.Y.; Palacios, A.E. Neural network burst pressure prediction in tall graphite-epoxy pressure vessels from acoustic emission data. JAE 2012, 30, 167-180.

12. Li, L.; Lomov, S.V.; Yan, X. Correlation of acoustic emission with optically observed damage in a glass/epoxy woven laminate under tensile loading, Compos Struct 2015, 123, 45-53, doi: 10.1016/j.compstruct.2014.12.029.

13. Xu, D.; Liu, P.F.; Chen, Z.P.; Leng, J.X.; Jiao, L. Achieving robust damage mode identification of adhesive composite joints for wind turbine blade using acoustic emission and machine learning. Compos Struct 2020, 236, article number 111840, doi: 10.1016/j.compstruct.2019.111840. 
14. Li, L.; Lomov, S.V.; Yan, X.; Carvelli, V. Cluster analysis of acoustic emission signals for 2D and 3D woven glass/epoxy composites. Compos Struct 2014, 116, 286-299, doi: 10.1016/j.compstruct.2014.05.023.

15. Bashkov, O.V.; Parfenov, E.E.; Bashkova, T.I. A soft hardware complex for recording and processing of acoustic signal and for location and indentification of their sources. Instrum Exp Tech 2010, 53(5), 682-687, doi: 10.1134/S0020441210050106.

16. Bryansky, A.A.; Bashkov, O.V.; Malysheva, D.P.; Solovev, D.B.. Investigation of the staging of damage accumulation in polymer composite materials during bending and tensile tests. Key Eng Mater 2021, 887, 116-122, doi: 10.4028/www.scientific.net/KEM.887.116.

17. Chandarana, N.; Ramasso, E.; Soutis, C.; Gresil, M. Damage identification in composites through acoustic emission monitoring. JAE 2019, 36, 91-96.

18. Chernov, D.; Elizarov, S.; Barat, V.; Vasilyev, I. Features of the AE testing of equipment on operating mode. JAE 2016, 33, 115124.

19. Qi, G.; Barhorst, A.; Hashemi, J.; Kamala, G. Discrete wavelet decomposition of acoustic emission signals from carbon-fiberreinforced composites. Compos Sci Technol 1997, 57(4), 389-403, doi: 10.1016/S0266-3538(96)00157-1

20. Bryansky, A.A.; Bashkov, O.V.; Bashkov, I.O.; Solovev, D.B. PCM bearing capacity prediction criteria development based on registered ae parameters. EEES 2020, 459(6), article number 062105, doi: 10.1088/1755-1315/459/6/062105.

21. Crivelli, D.; Guagliano, M.; Eaton, M.; Pearson, M.; Al-Jumaili, S.; Holford, K.; Pullin, R. Localisation and identification of fatigue matrix cracking and delamination in a carbon fibre panel by acoustic emission. Compos B Eng 2015, 74, 1-12, doi: 10.1016/j.compositesb.2014.12.032.

22. Heslehurst, R.B. Defects and Damage in Composite Materials and Structures; CRC press: Boca Raton, Florida, $2014 ;$ pp. 79-101.

23. Maillet, E.; Baker, C.; Morscher, G.N.; Pujar, V.V.; Lemanski, J.R. Feasibility and limitations of damage identification in composite materials using acoustic emission. Compos - A: Appl Sci Manuf 2015, 75, 77-83, doi: 10.1016/j.compositesa.2015.05.003.

24. Ciampa, F.; Meo, M. A new algorithm for acoustic emission localization and flexural group velocity determination in anisotropic structures. Compos - A: Appl Sci Manuf 2010, 41(12), 1777-1786, doi: 0.1016/j.compositesa.2010.08.013.

25. Niri, E.D.; Farhidzadeh, A.; Salamone, S. Nonlinear Kalman Filtering for acoustic emission source localization in anisotropic panels. Ultrasonics 2014, 54(2), 486-501, doi: 10.1016/j.ultras.2013.07.016.

26. Sikdar, S.; Mirgal, P.; Banerjee, S.; Ostachowicz, W. Damage-induced acoustic emission source monitoring in a honeycomb sandwich composite structure. Compos B Eng 2019, 158, 179-188, doi: 10.1016/j.compositesb.2018.09.071.

27. Siron, O.; Tsuda, H. Acoustic emission in carbon fibre-reinforced plastic materials. Ann Chim - Sci Mat 2000, 25(7), 533-537, doi: 10.1016/S0151-9107(01)80006-4.

28. Giordano, M.; Calabro, A.; Esposito, C.; D'amore, A.; Nicolais, L. An acoustic-emission characterization of the failure modes in polymer-composite materials. Compos Sci Technol 1998, 58(12), 1923-1928, doi: 10.1016/S0266-3538(98)00013-X.

29. Asamene, K.; Hudson, L.; Sundaresan, M. Influence of attenuation on acoustic emission signals in carbon fiber reinforced polymer panels. Ultrasonics 2015, 59, 86-93, doi: 10.1016/j.ultras.2015.01.016.

30. Hamam, Z.; Godin, N.; Fusco, C.; Monnier, T. Characterization of fiber break by quantitative acoustic emission in a model carbon/epoxy composite. JAE 2019, 36, S85-S90.

31. Liang, S.; Zhang, C.; Lin, W.; Li, L.; Li, C.; Feng, X.; Lin, B. Fiber-optic intrinsic distributed acoustic emission sensor for large structure health monitoring. Optics Letters 2009, 34(12), 1858-1860, doi: 10.1364/OL.34.001858.

32. Fu, T.; Liu, Y.; Li, Q.; Leng, J. Fiber optic acoustic emission sensor and its applications in the structural health monitoring of CFRP materials. Opt Lasers Eng 2009, 47(10), 1056-1062, doi: 10.1016/j.optlaseng.2009.03.011.

33. Kulchin, Y. N.; Vitrik, O. B.; Romashko, R. V.; Petrov, Y. S.; Kirichenko, O. V.; Kamenev, O. T. Tomography methods for vector field study using space-distributed fiber optic sensors with integral sensitivity. Fiber and Integrated Optics 1998, 17(1), 75-84.

34. Bashkov, O.V.; Romashko, R.V.; Zaikov, V.I.; Panin, S.V.; Bezruk, M.N.; Khun, K.; Bashkov, I.O.. Detecting acoustic-emission signals with fiber-optic interference transducers. Russ J Nondestruct Test 2017, 53(6), 415-421, doi: 10.1134/S1061830917060031.

35. Bashkov, O.V.; Romashko, R.V.; Khon, H.; Bezruk, M.N.; Zaikov, V.I.; Bashkov, I.O. Registration of acoustic emission waves in anisotropic composite plates by fiber-optic sensors. Proceedings of SPIE, 2017, 11024, 110240O, doi: 10.1117/12.2518272.

36. Wei, P.; Han, X.; Xia, D.; Liu, T.; Lang, H. Novel fiber-optic ring acoustic emission sensor. Sensors 2018, 18(1), article number 215, doi: 10.3390/s18010215.

37. Kamshilin, A. A.; Romashko, R. V.; Kulchin, Y. N. Adaptive interferometry with photorefractive crystals. J Applied Physics 2009, 105(3), 031101.

38. Di Girolamo, S.; Romashko, R. V.; Kulchin, Y. N.; Kamshilin, A. A. Orthogonal geometry of wave interaction in a photorefractive crystal for linear phase demodulation. Optics Communications 2010, 283(1), 128-131.

39. Romashko, R. V.; Bezruk, M. N.; Kamshilin, A. A.; Kulchin, Y. N. Six-channel adaptive fibre-optic interferometer. Quantum Electronics 2012, 42(6), 551.

40. Romashko, R.; Bezruk, M.; Ermolaev, S.; Storozhenko, D.; Kulchin, Y. Adaptive Distributed Fiber-Optical Sensory Network For Detecting Weak Acoustic Fields. In Advanced Photonics 2016 (IPR, NOMA, Sensors, Networks, SPPCom, SOF), OSA Technical Digest (online); Vancouver, Canada, 18-20 July 2016; Optical Society of America, 2016; paper JTu4A.23. 